Supporting Information for

\title{
Enhancing the Anode Performance of Antimony through Nitrogen-Doped Carbon and Carbon Nanotubes
}

Xia Liu, Yichen Du, Xin Xu, Xiaosi Zhou, * Zhihui Dai, * and Jianchun Bao

Jiangsu Key Laboratory of Biofunctional Materials, School of Chemistry and Materials

Science, Nanjing Normal University, Nanjing 210023, P. R. China

*(X.Z.) E-mail: zhouxiaosi@njnu.edu.cn; Telephone/Fax: +86-25-85891027.

*(Z.D.) E-mail: daizhihuii@njnu.edu.cn; Telephone/Fax: +86-25-85891051. 


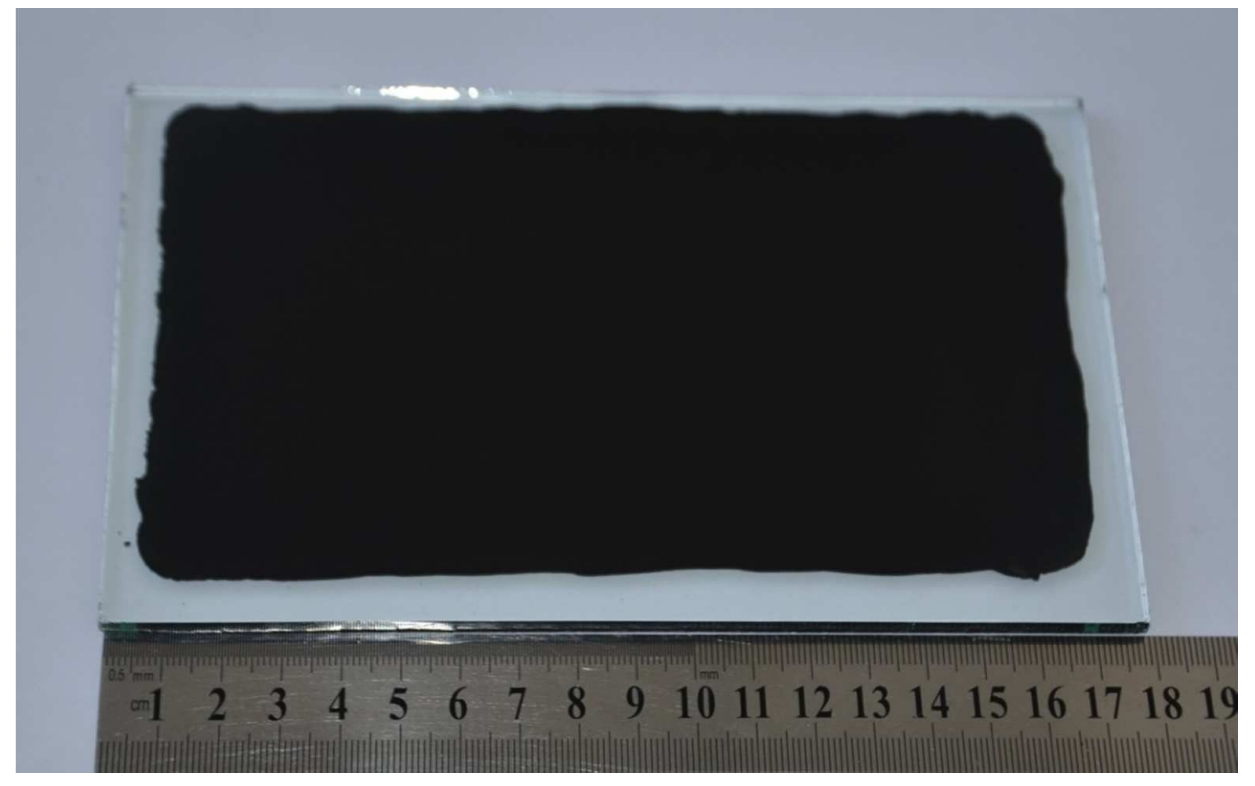

Figure S1. Photograph of the Sb/PAN+CNTs film. 


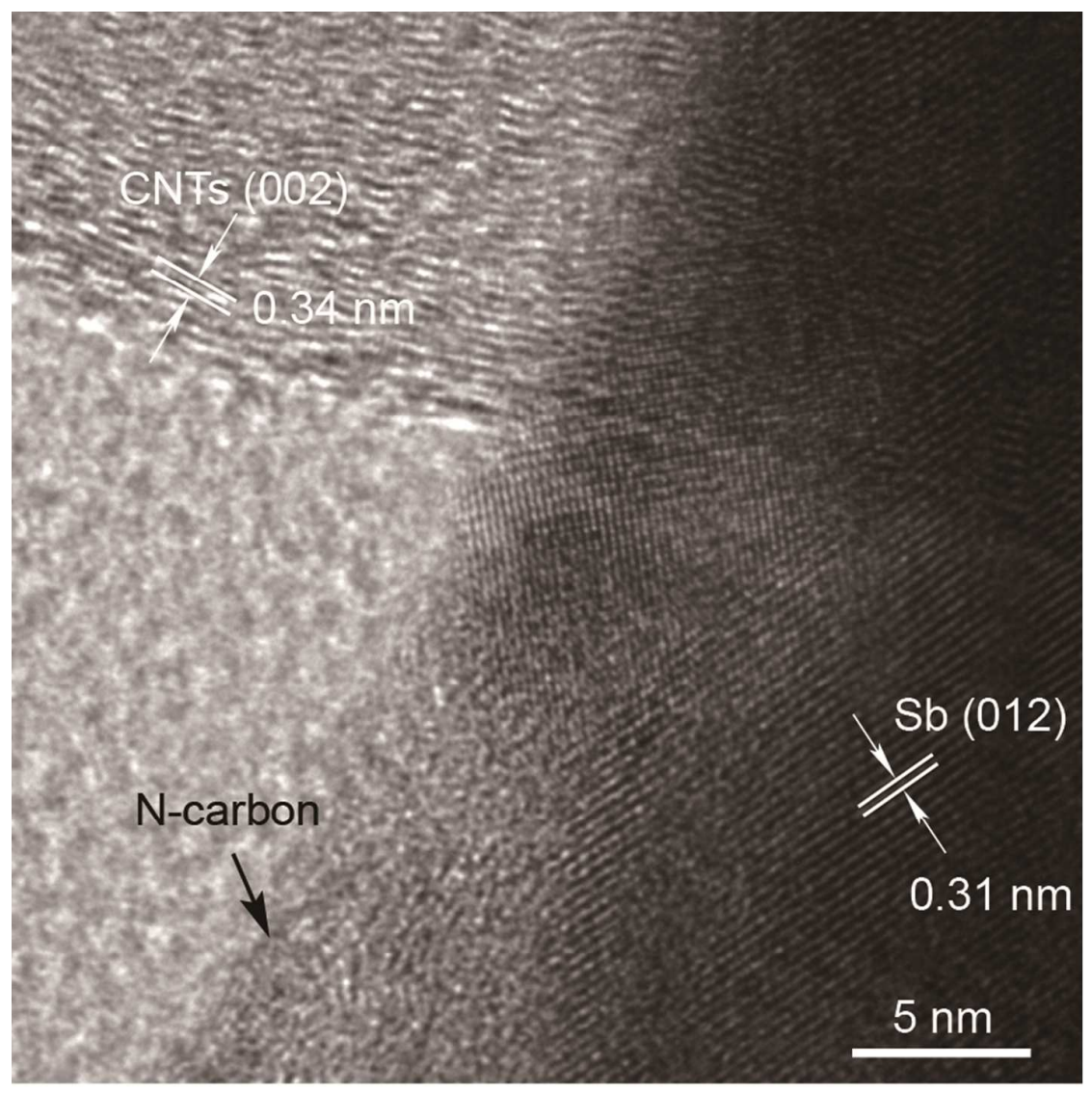

Figure S2. HRTEM image of Sb/N-carbon+CNTs. 


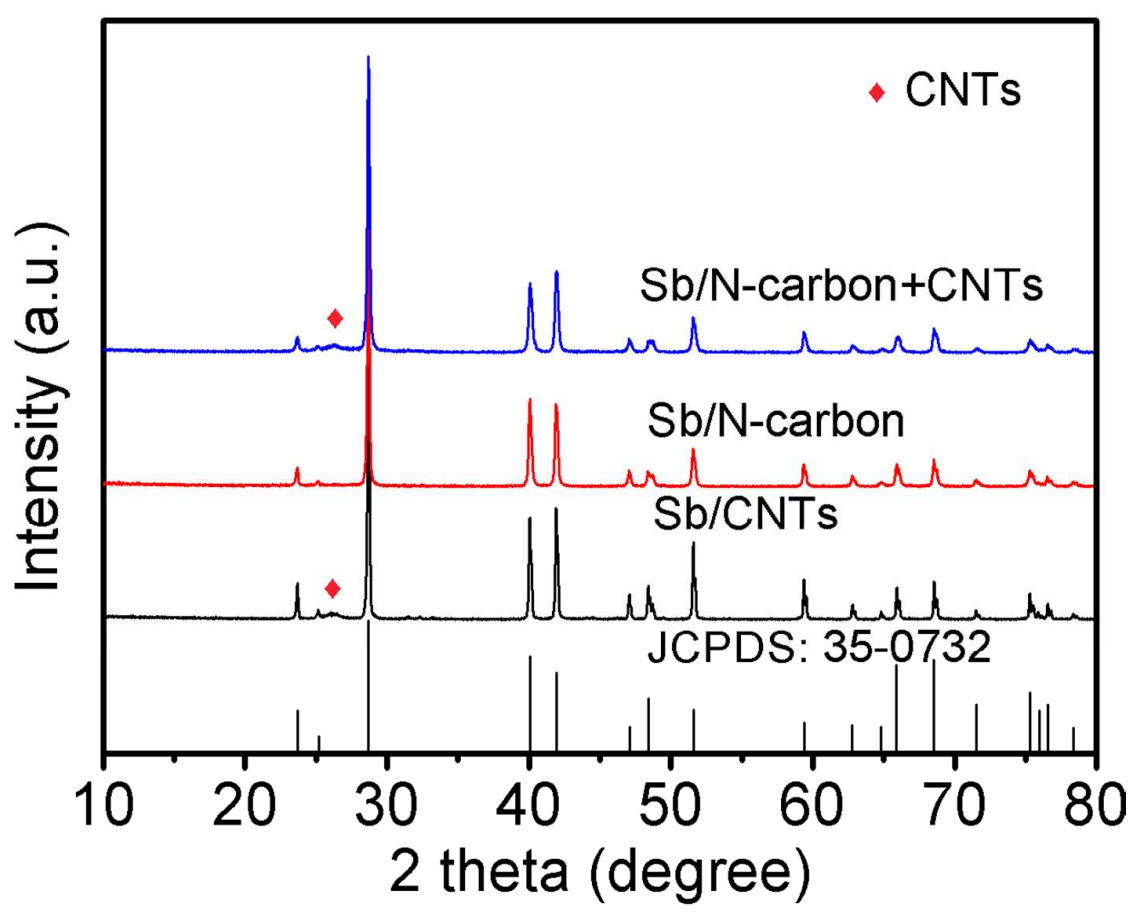

Figure S3. XRD patterns of the $\mathrm{Sb} / \mathrm{CNTs}, \mathrm{Sb} / \mathrm{N}$-carbon, and $\mathrm{Sb} / \mathrm{N}$-carbon $+\mathrm{CNTs}$ composites and standard XRD pattern of Sb (JCPDS card No. 35-0732). 

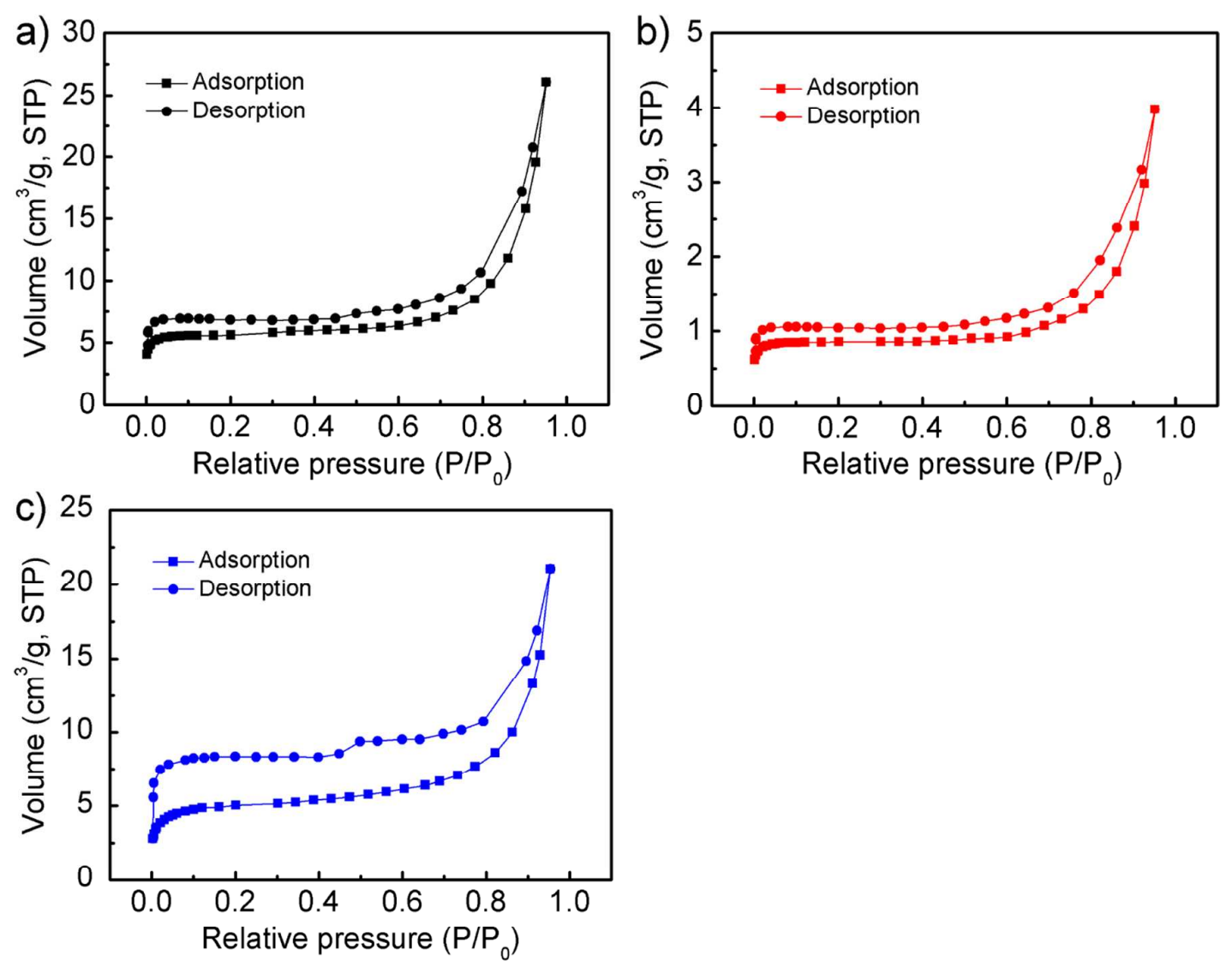

Figure S4. Nitrogen adsorption/desorption isotherms of (a) Sb/CNTs, (b) Sb/N-carbon, and (c) $\mathrm{Sb} / \mathrm{N}$-carbon+CNTs and their corresponding BET surface area are $20.9,4.2$, and $16.5 \mathrm{~m}^{2}$ $\mathrm{g}^{-1}$, respectively. 


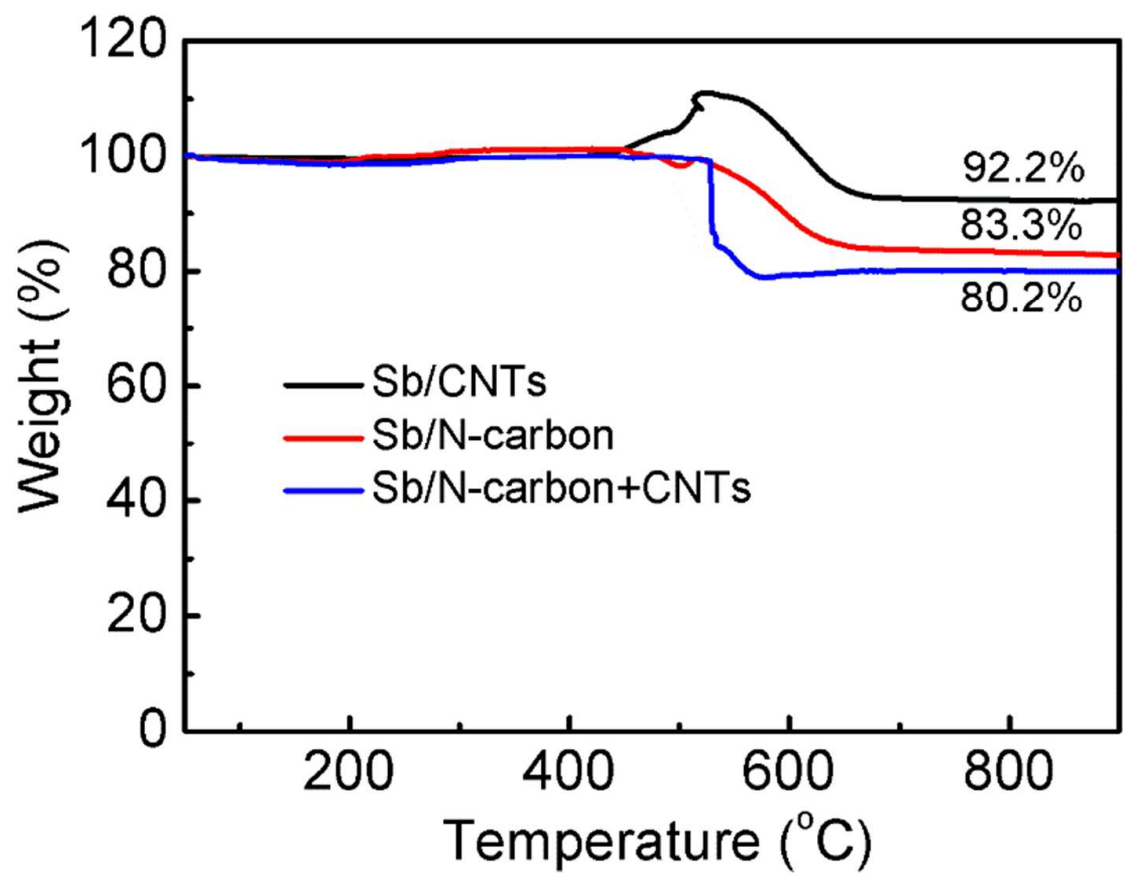

Figure S5. TGA curves of $\mathrm{Sb} / \mathrm{CNTs}, \mathrm{Sb} / \mathrm{N}$-carbon, and $\mathrm{Sb} / \mathrm{N}$-carbon+CNTs in air atmosphere with a heating rate of $10{ }^{\circ} \mathrm{C} \min ^{-1}$. 

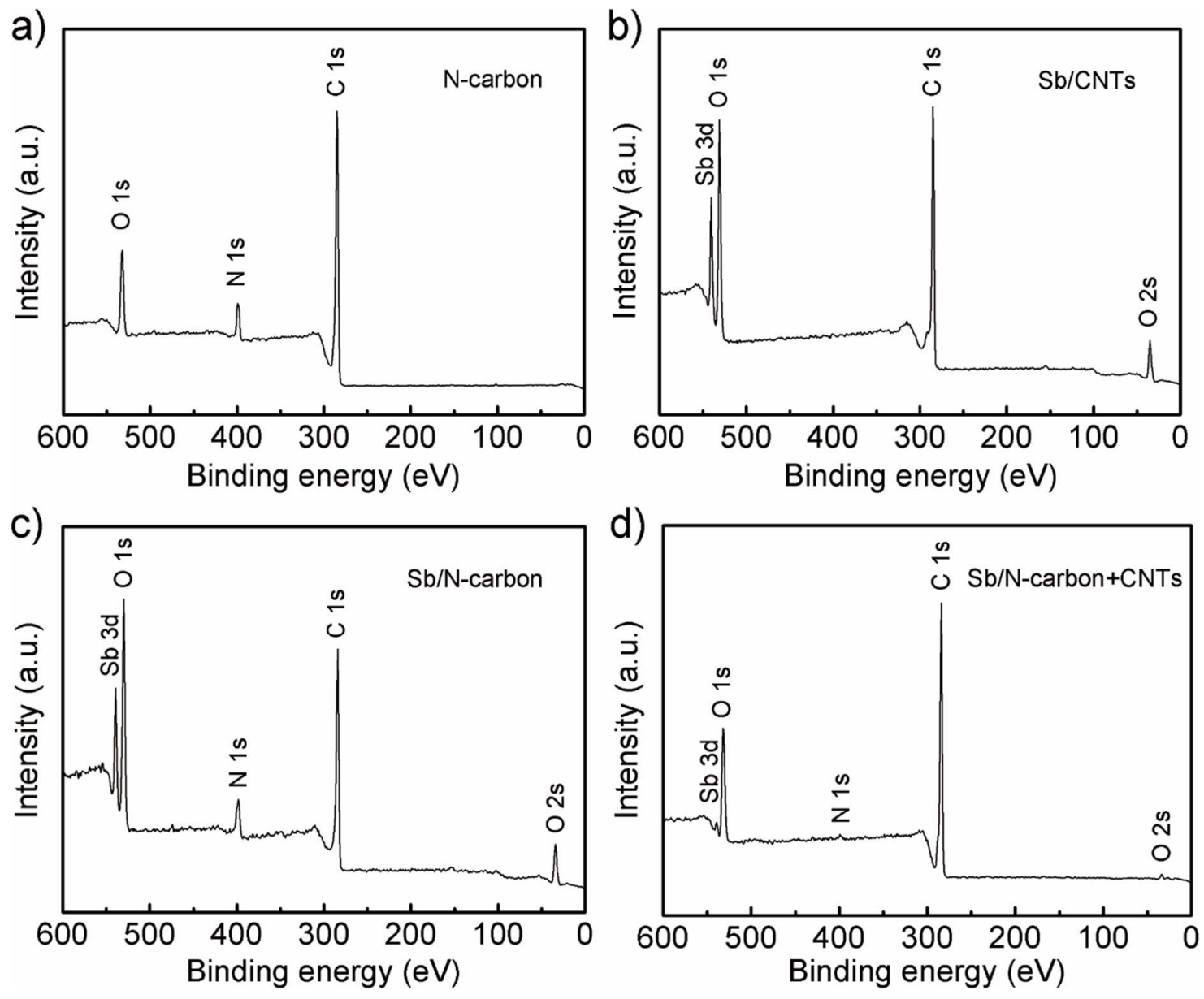

Figure S6. XPS survey scans of (a) N-carbon, (b) Sb/CNTs, (c) Sb/N-carbon, and (d) $\mathrm{Sb} / \mathrm{N}$-carbon+CNTs. The high-resolution N 1s XPS spectrum is shown in Figure $3 \mathrm{~b}$. 


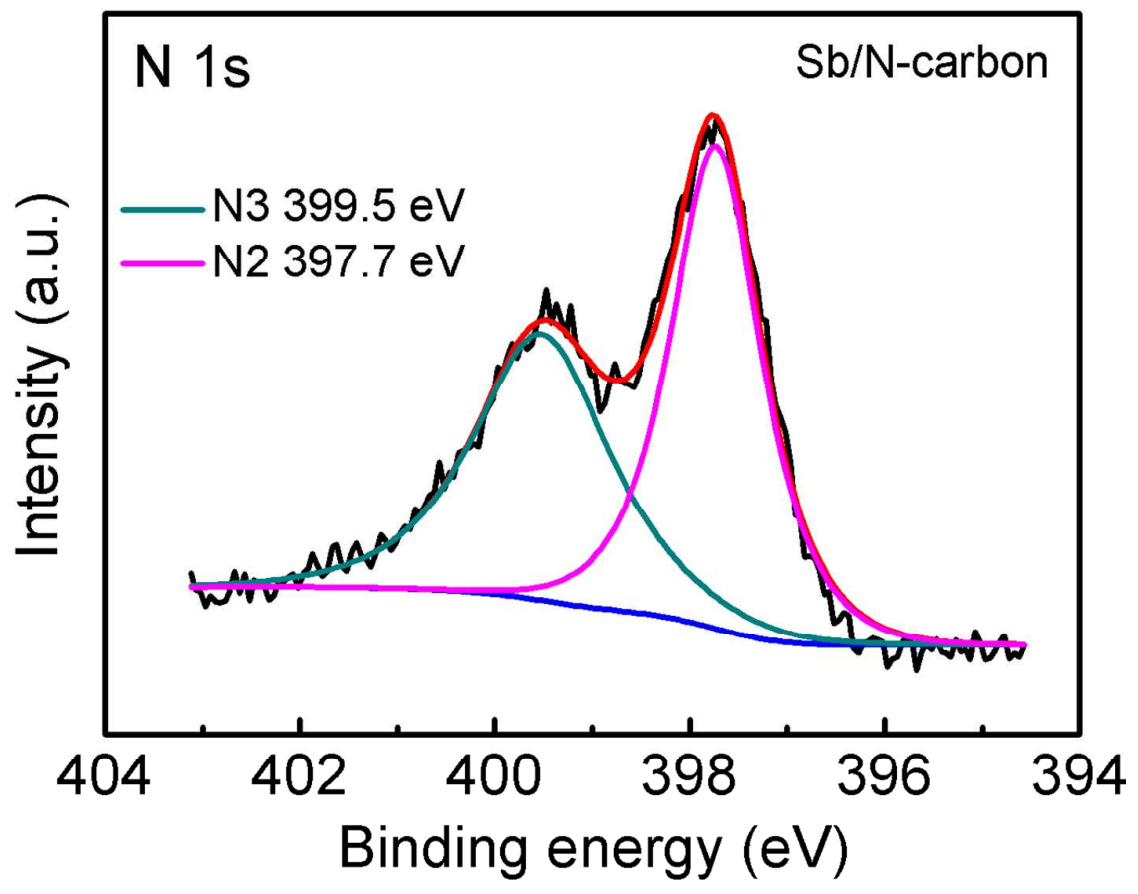

Figure S7. High-resolution N 1s XPS spectum of Sb/N-carbon. 


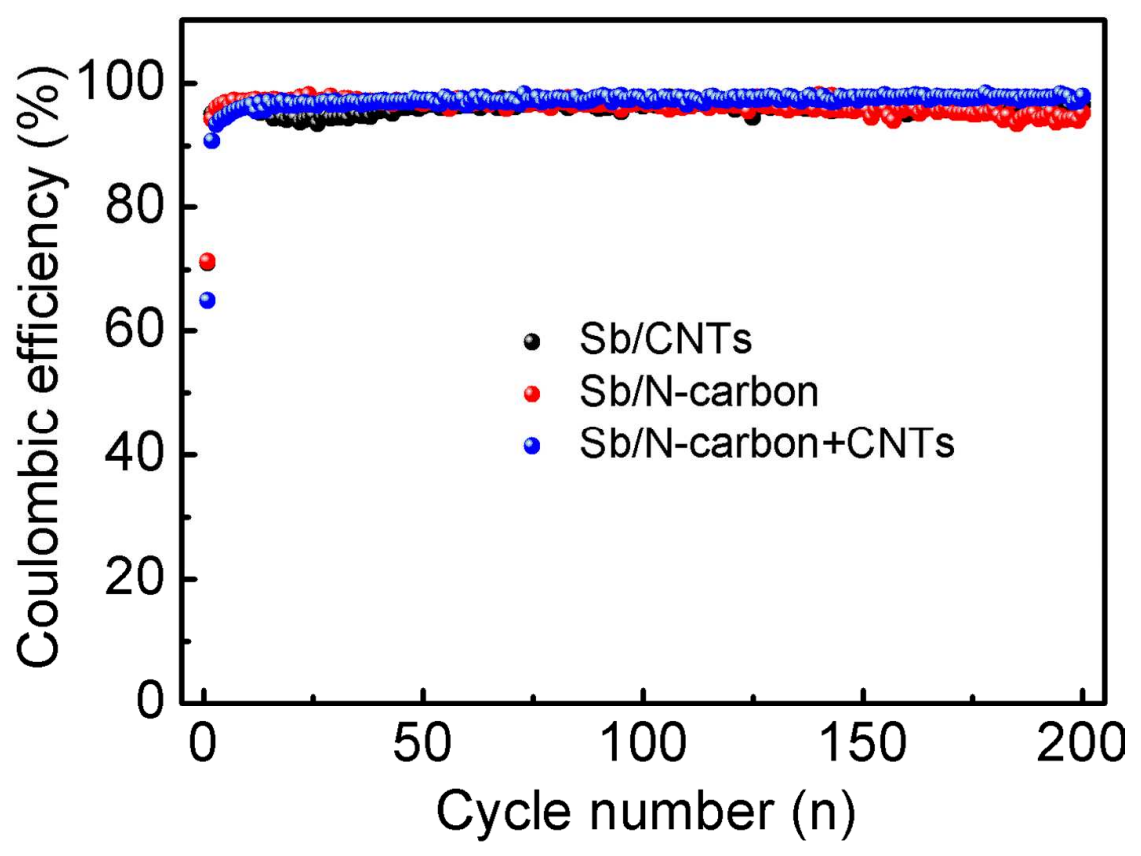

Figure S8. Coulombic efficiencies of $\mathrm{Sb} / \mathrm{CNTs}, \mathrm{Sb} / \mathrm{N}$-carbon, and $\mathrm{Sb} / \mathrm{N}$-carbon $+\mathrm{CNTs}$ at a current density of $0.1 \mathrm{~A} \mathrm{~g}^{-1}$ for 200 cycles. 
Table S1. Electrochemical performances of various kinds of Sb-based anode materials for sodium-ion batteries.

\begin{tabular}{|c|c|c|c|c|c|c|}
\hline Materials & $\begin{array}{c}\text { Ratio of active } \\
\text { material } \\
\text { (wt \%) }\end{array}$ & $\begin{array}{l}\text { Voltage range } \\
\left(\mathrm{V} \mathrm{vs} \mathrm{Na}^{+} / \mathrm{Na}\right)\end{array}$ & $\begin{array}{l}\text { Mass loading } \\
\left(\mathbf{m g} / \mathbf{c m}^{2}\right)\end{array}$ & $\begin{array}{l}\text { Current } \\
\text { density } \\
(\mathrm{mA} / \mathrm{g})\end{array}$ & $\begin{array}{l}\text { Charge } \\
\text { capacity } \\
(\mathrm{mA} / \mathrm{h} \text { g) }\end{array}$ & Reference \\
\hline $\mathrm{Sb} / \mathrm{N}$-carbon $+\mathrm{CNTs}$ & 70 & $0.01-2$ & $1.0-1.2$ & 100 & $\begin{array}{c}476 \text { (after } 200 \\
\text { cycles) }\end{array}$ & This work \\
\hline $\mathrm{Sb}-\mathrm{C}$ nanofibers & 70 & $0.01-2.5$ & $\sim 2.5$ & 200 & $\begin{array}{c}446 \text { (after } 400 \\
\text { cycles) }\end{array}$ & S1 \\
\hline SbNP@C & 100 & $0-2$ & $\sim 3 \mathrm{mg}$ & 100 & $\begin{array}{c}350 \text { (after } 300 \\
\text { cycles) }\end{array}$ & S2 \\
\hline $\begin{array}{c}\mathrm{Sb} / \mathrm{MWCNT} \\
\text { nanocomposite }\end{array}$ & 80 & $0-2$ & $1.2-1.5$ & 200 & $\begin{array}{c}382 \text { (after } 120 \\
\text { cycles) }\end{array}$ & $\mathrm{S} 3$ \\
\hline $\mathrm{Sb} / \mathrm{ICNNs}$ & 70 & $0.01-2$ & Not reported & 100 & $\begin{array}{c}525 \text { (after } 100 \\
\text { cycles) }\end{array}$ & S4 \\
\hline $\mathrm{Sb}-\mathrm{N} / \mathrm{C}$ & 75 & $0.01-3.00$ & $1.0-2.0 \mathrm{mg}$ & 50 & $\begin{array}{c}305 \text { (after } 60 \\
\text { cycles) }\end{array}$ & S5 \\
\hline $\begin{array}{l}\mathrm{Sb}-\text { carbon } \\
\text { composite } \\
\text { microspheres }\end{array}$ & 70 & $0.001-2$ & Not reported & 300 & $\begin{array}{c}372 \text { (after } 100 \\
\text { cycles) }\end{array}$ & S6 \\
\hline $\mathrm{Sb} / \mathrm{AB}$ composites & 70 & $0.01-2$ & $1.0-1.2 \mathrm{mg}$ & 100 & $\begin{array}{c}473 \text { (after } 70 \\
\text { cycles) }\end{array}$ & S7 \\
\hline $\mathrm{Sb} / \mathrm{G}$ & 75 & $0.005-1.5$ & Not reported & 20 & $\begin{array}{c}211 \text { (after } 30 \\
\text { cycles) }\end{array}$ & S8 \\
\hline $\mathrm{rGO} / \mathrm{Sb}$ & 80 & $0.01-1.5$ & $1.02-1.05$ & 131 & $\begin{array}{c}598 \text { (after } 50 \\
\text { cycles) }\end{array}$ & S9 \\
\hline 10-Sb@C & 80 & $0.01-2.0$ & $\sim 0.8$ & 100 & $\begin{array}{c}385 \text { (after } 500 \\
\text { cycles) }\end{array}$ & S10 \\
\hline $\begin{array}{c}\mathrm{SiC}-\mathrm{Sb}-\mathrm{C} \\
\text { nanocomposites }\end{array}$ & 70 & $0.01-2.0$ & Not reported & 100 & $\begin{array}{c}595 \text { (after } 100 \\
\text { cycles) }\end{array}$ & S11 \\
\hline $\mathrm{Sb} / \mathrm{MLG}$ hybrid & 80 & $0.01-2$ & $1.0-1.5$ & 100 & $\begin{array}{c}405 \text { (after } 200 \\
\text { cycles) }\end{array}$ & S12 \\
\hline $\begin{array}{l}\text { Pure micrometric } \\
\text { antimony }\end{array}$ & 70 & $0.01-1.5$ & 1.4 & $\mathrm{C} / 2$ & $\begin{array}{c}576 \text { (after } 160 \\
\text { cycles) }\end{array}$ & S13 \\
\hline $\begin{array}{c}\mathrm{Sb} / \mathrm{C} \\
\text { nanocomposites }\end{array}$ & 80 & $0-2$ & 3 & 100 & $\begin{array}{c}580 \text { (after } 100 \\
\text { cycles) }\end{array}$ & S14 \\
\hline $\mathrm{Sb} \mathrm{NCs}$ & 64 & $0-1.5$ & $0.5^{-1}$ & 660 & $\begin{array}{l}\sim 580 \text { (after } \\
100 \text { cycles) }\end{array}$ & S15 \\
\hline Sb HNSs & 70 & $0.01-2$ & Not reported & 50 & $\begin{array}{c}622 \text { (after } 50 \\
\text { cycles) }\end{array}$ & S16 \\
\hline $\begin{array}{c}\mathrm{Sb} @ \mathrm{C} \\
\text { microspheres }\end{array}$ & 80 & $0-2.0$ & Not reported & 200 & $\begin{array}{c}584 \text { (after } 100 \\
\text { cycles) }\end{array}$ & S17 \\
\hline
\end{tabular}




\begin{tabular}{|c|c|c|c|c|c|c|}
\hline $\mathrm{Sb}$ PHMSs & 70 & $0.01-2$ & $1.0-1.2 \mathrm{mg}$ & 100 & $\begin{array}{c}600 \text { (after } 100 \\
\text { cycles) }\end{array}$ & $\mathrm{S} 18$ \\
\hline
\end{tabular}

Note that the charge capacities are calculated on the basis of the total mass of the composites except Ref. S13-18, in which the capacity values are normalized by the mass of Sb alone.

\section{References}

(S1) Wu, L.; Hu, X.; Qian, J.; Pei, F.; Wu, F.; Mao, R.; Ai, X.; Yang, H.; Cao, Y. Sb-C Nanofibers with Long Cycle Life as an Anode Material for High-Performance Sodium-Ion Batteries. Energy Environ. Sci. 2014, 7, 323-328.

(S2) Zhu, Y.; Han, X.; Xu, Y.; Liu, Y.; Zheng, S.; Xu, K.; Hu, L.; Wang, C. Electrospun Sb/C Fibers for a Stable and Fast Sodium-Ion Battery Anode. ACS Nano 2013, 7, 6378-6386.

(S3) Zhou, X.; Dai, Z.; Bao, J.; Guo, Y.-G. Wet Milled Synthesis of an Sb/MWCNT Nanocomposite for Improved Sodium Storage. J. Mater. Chem. A 2013, 1, 13727-13731.

(S4) Hou, H.; Jing, M.; Yang, Y.; Zhang, Y.; Song, W.; Yang, X.; Chen, J.; Ji. X. Antimony Nanoparticles Anchored on Interconnected Carbon Nanofibers Networks as Advanced Anode Material for Sodium-Ion Batteries. J. Power Sources 2015, 284, 227-235.

(S5) Zhou, X.; Zhong, Y.; Yang, M.; Hu, M.; Wei, J.; Zhou, Z. Sb Nanoparticles Decorated N-Rich Carbon Nanosheets as Anode Materials for Sodium Ion Batteries with Superior Rate Capability and Long Cycling Stability. Chem. Commun. 2014, 50, 12888-12891.

(S6) Ko, Y. N.; Kang, Y. C. Electrochemical Properties of Ultrafine Sb Nanocrystals Embedded in Carbon Microspheres for Use as Na-Ion Battery Anode Materials. Chem. Commun. 2014, 50, 12322-12324.

(S7) Hou, H.; Yang, Y.; Zhu, Y.; Jing, M.; Pan, C.; Fang, L.; Song, W.; Yang, X.; Ji, X. An Electrochemical Study of Sb/Acetylene Black Composite as Anode for Sodium-Ion Batteries. Electrochim. Acta 2014, 146, 328-334.

(S8) Zhang, Y.; Xie, J.; Zhu, T.; Cao, G.; Zhao, X.; Zhang, S. Activation of Electrochemical Lithium and Sodium Storage of Nanocrystalline Antimony by Anchoring on Graphene via a Facile In Situ Solvothermal Route. J. Power Sources 2014, 247, 204-212.

(S9) Nithya, C.; Gopukumar, S. rGO/Nano Sb Composite: A High Performance Anode 
Material for $\mathrm{Na}^{+}$Ion Batteries and Evidence for the Formation of Nanoribbons from the Nano rGO Sheet during Galvanostatic Cycling. J. Mater. Chem. A 2014, 2, 10516-10525.

(S10) Zhang, N.; Liu, Y.; Lu, Y.; Han, X.; Cheng, F.; Chen, J. Spherical Nano-Sb@C Composite as a High-Rate and Ultra-Stable Anode Material for Sodium-Ion Batteries. Nano Res. 2015, 8, 3384-3393.

(S11) Wu, L.; Pei, F.; Mao, R.; Wu, F.; Wu, Y.; Qian, J.; Cao, Y.; Ai, X.; Yang, H. SiC-Sb-C Nanocomposites as High-Capacity and Cycling-Stable Anode for Sodium-Ion Batteries. Electrochim. Acta 2013, 87, 41-45.

(S12) Hu, L.; Zhu, X.; Du, Y.; Li, Y.; Zhou, X.; Bao, J. A Chemically Coupled Antimony/Multilayer Graphene Hybrid as a High-Performance Anode for Sodium-Ion Batteries. Chem. Mater. 2015, 27, 8138-8145.

(S13) Darwiche, A.; Marino, C.; Sougrati, M. T.; Fraisse, B.; Stievano, L.; Monconduit, L. Better Cycling Performances of Bulk Sb in Na-Ion Batteries Compared to Li-Ion Systems: An Unexpected Electrochemical Mechanism. J. Am. Chem. Soc. 2012, 134, 20805-20811.

(S14) Qian, J.; Chen, Y.; Wu, L.; Cao, Y.; Ai, X.; Yang, H. High Capacity Na-Storage and Superior Cyclability of Nanocomposite Sb/C Anode for Na-Ion Batteries. Chem. Commun. 2012, 48, 7070-7072.

(S15) He, M.; Kraychyk, K.; Walter, M.; Kovalenko, M. V. Monodisperse Antimony Nanocrystals for High-Rate Li-ion and Na-ion Battery Anodes: Nano versus Bulk. Nano Lett. 2014, 14, 1255-1262.

(S16) Hou, H.; Jing, M.; Yang, Y.; Zhu, Y.; Fang, L.; Song, W.; Pan, X.; Ji, X. Sodium/Lithium Storage Behavior of Antimony Hollow Nanospheres for Rechargeable Batteries. ACS Appl. Mater. Interfaces 2014, 6, 16189-16196.

(S17) Wu, L.; Lu, H.; Xiao, L.; Ai, X.; Yang, H.; Cao, Y. Electrochemical Properties and Morphological Evolution of Pitaya-Like Sb@C Microspheres as High-Performance Anode for Sodium Ion Batteries. J. Mater. Chem. A 2015, 3, 5708-5713.

(S18) Hou, H.; Jing, M.; Yang, Y.; Zhang, Y.; Zhu, Y.; Song, W.; Yang, X.; Ji, X. Sb Porous Hollow Microspheres as Advanced Anode Materials for Sodium-Ion Batteries. J. Mater. Chem. A 2015, 3, 2971-2977. 